\title{
Short-Term Effect of Topical Cetylated Fatty Acid on Early and Advanced Knee Osteoarthritis: A Multi-Center Study
}

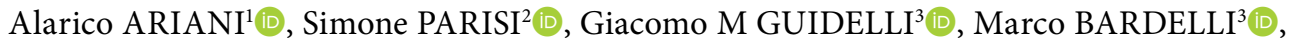 \\ Augusto BERTINI ${ }^{4}$, Enrico FUSARO2 ${ }^{2}$ \\ ${ }^{1}$ Department of Medicine Internal Medicine and Rheumatology Unit, Azienda Ospedaliero - Universitaria Di Parma, Parma, Italy \\ ${ }^{2}$ Department Rheumatology, Azienda Ospedaliera Cittá Della Salute E Della Scienza Di Torino, Torino, Italy \\ ${ }^{3}$ Department of Medicine, Surgery and Neurosciences, Rheumatology Unit, Siena University Hospital, Siena, Italy \\ ${ }^{4}$ Azienda Unità Sanitaria Locale Di Parma, South-east District, Polo Di Langhirano, Parma, Italy
}

\begin{abstract}
Objectives: This study aims to investigate if short-term topical treatment with cetylated fatty acid (CFA) cream reduces the detrimental effects of early and advanced knee osteoarthritis (OA).

Patients and methods: The study included 113 patients (32 males, 81 females; median age 70.0 years; 95\% Cl: 69.0 to 71.4 years) with knee OA diagnosed according to American College of Rheumatology classification criteria. Each patient underwent knee X-rays, followed by a CFA topical treatment (two applications per day for one week). Before and after the treatment, patients completed a Western Ontario and McMaster Universities Osteoarthritis Index (WOMAC) questionnaire. All knee X-rays were classified according to Kellgren-Lawrence (K-L) scale.

Results: After one week of treatment, decreased WOMAC overall scores and sub-scale scores were observed in the whole cohort $(p<0.005)$ and $\mathrm{K}-\mathrm{L}$ scale grade 3 group $(\mathrm{p}<0.05)$. In the K-L scale grade 2 group, overall WOMAC scores and pain and functional ability sub-scale scores improved $(p<0.05)$.

Conclusion: Administration of topical CFA may mitigate most common symptoms in knee OA. Our findings suggest that topical CFA is effective in all knee OA patients with slightly higher evidence for those with advanced disease.

Keywords: Cetylated fatty acid; cutaneous drug administration; knee osteoarthritis.
\end{abstract}

Knee osteoarthritis (OA) is one of the most common degenerative affections of the lower extremities. Its prevalence ranges from 5 to $25 \%$ in males and from 5 to $40 \%$ in females in 40 to 80-year-old subjects. ${ }^{1,2}$ As incidence of knee $\mathrm{OA}$ increases with age, it appears to be higher in females $\left(1.4-2.0 \%\right.$ vs. $2.2-3.7 \%$ per year). ${ }^{3}$ who are also burdened with a considerable lifetime risk of symptomatic knee OA by the age of 85 $(46.8 \% \text { vs. } 39.8 \%)^{1}$
Guidelines from the Osteoarthritis Research Society International and American Academy of Orthopaedic Surgeons on non-surgical management of knee OA.,5 recommend non-pharmacological strategies (such as the use of walking cane, land- or water-based exercises, and biomechanical interventions) and drugs. The latter may be oral medications (paracetamol, duloxetine, and non-steroidal anti-inflammatory drugs [NSAIDs]), injections

Received: November 02, 2017 Accepted: January 15, 2018 Published online: March 23, 2018

Correspondence: Alarico Ariani, MD. Department of Medicine Internal Medicine and Rheumatology Unit, Azienda Ospedaliero - Universitaria Di Parma, 43125 Parma, Italy. Tel: +390521 702776 e-mail: dott.alaricoariani@libero.it

\section{Citation:}

Ariani A, Parisi S, Guidelli GM, Bardelli M, Bertini A, Fusaro E. Short-term effect of topical cetylated fatty acid on early and advanced knee osteoarthritis: A multi-center study. Arch Rheumatol 2018;33(4):438-442. 
(corticosteroids, hyaluronic acids), and topical creams (NSAIDs, capsaicin). Although topical treatment may present less side effects compared to oral medications, it may still cause adverse events leading to withdrawal. Indeed, the cardiovascular risk related to NSAIDs seems to further increase subchondral bone ischemia hastening the $\mathrm{OA}$ progression. ${ }^{6}$

Oral and topical cetylated fatty acids (CFAs) are not known to have any adverse or unwanted effects while their benefits have been well-demonstrated; however, their mechanism of action has not been well-elucidated. Reduced pro-inflammatory cytokine levels, changes in membrane fluidity and signal transduction pathways reducing the proteoglycan degrading enzyme activity, and release of leukotriene B4 from neutrophils have been hypothesized. ${ }^{7,8}$

Cetylated fatty acids proved to be helpful in knee, elbow, and wrist OA as well as in myofascial pain syndrome of the neck..$^{8-10}$ In particular, CFA topical treatment in knee OA subjects improved movement range, the ability to ascend and descend stairs, and to rise from sitting., ${ }^{9,11,12}$

Using X-rays, knee OA can be classified into the following grades: minimal (early knee OA), moderate (advanced knee OA), and severe (late knee OA) ${ }^{13}$ In the first two stages, surgical knee replacement using prosthesis is avoidable and the use of systemic analgesics or pharmacological topical treatment is common. However, to our knowledge, the stage of knee OA where CFA is most effective has not been assessed. Therefore, in this study, we aimed to investigate if short-term topical treatment with CFA cream reduces the detrimental effects of early and advanced knee OA.

\section{PATIENTS AND METHODS}

One-hundred and thirteen consecutive knee OA patients (32 males, 81 females; median age 70.0 years; 95\% CI: 69.0 to 71.4 years) from four centers were enrolled between January 2014 and December 2016. Diagnosis was established according to American College of Rheumatology (ACR) and European League Against Rheumatism (EULAR) classification criteria. ${ }^{14}$ Exclusion criteria were age below 18 years, concomitant knee arthritis, previous knee fractures or major trauma,

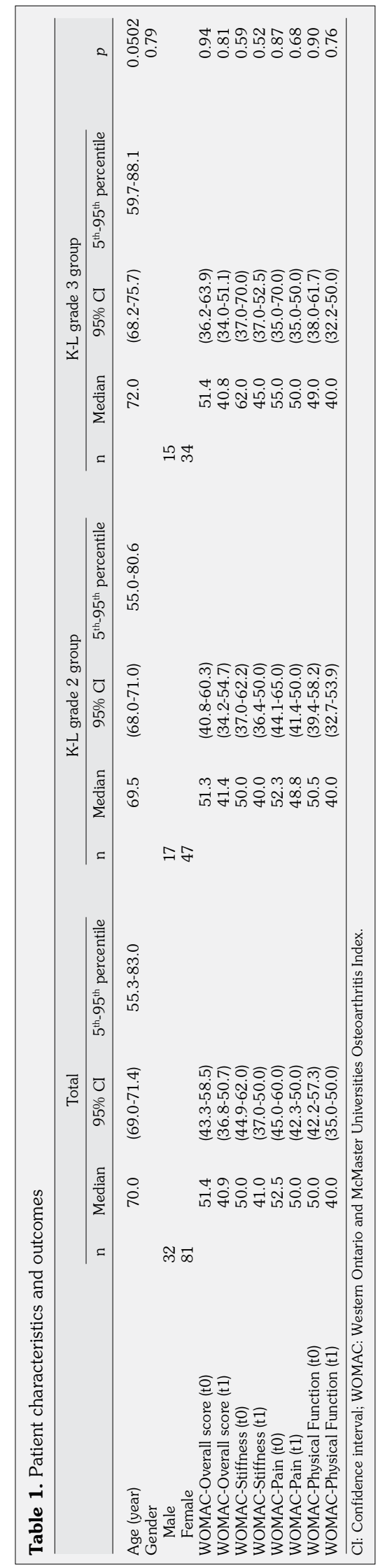


body weight increase or decrease $( \pm 5 \mathrm{~kg})$, and relevant change of activity level during the period of the study. Only one knee was assessed for each patient. Analgesics (such as paracetamol and opioids) were permitted but patients could not exceed the dosages taken before the study beginning. All patients used a CFA-based topical cream mainly made of a blend of CFA (Cetilar ${ }^{\circledR}$, PharmaNutra, Pisa, Italy). Each patient was instructed to apply a standard quantity of cream on the most painful knee twice per day during a period of one week. The study protocol was approved by the Local Ethics Committees. A written informed consent was obtained from each patient. The study was conducted in accordance with the principles of the Declaration of Helsinki.

The most painful knee of each patient was assessed with an anteroposterior X-ray before the CFA treatment. Each X-ray was evaluated according to the Kellgren-Lawrence (K-L) scale: ${ }^{13}$ grades 2 and 3 were considered as expression of early and advanced knee OA, respectively.

The clinical spectrum of knee OA was measured using the Western Ontario and McMaster Universities Osteoarthritis Index (WOMAC). The analysis of WOMAC sub-scales gives an accurate insight of the most common OA signs and symptoms (stiffness, pain, and functional limitation). The Italian version of WOMAC questionnaire ${ }^{15}$ was administrated to each patient twice: the first time one to five days before the beginning of CFA local application (baseline or $\mathrm{t} 0$ ) and the second time after three days from the end of CFA treatment (ending or $\mathrm{t} 1$ ).

\section{Statistical analysis}

Statistical analysis was performed using $\mathrm{R}$ software (version 3.3.2, www.r-project.org, last visit on 11/01/2017). Non-parametric tests (MannWhitney U test and chi-squared test) as appropriate were used to compare subgroup characteristics (age, sex, and WOMAC overall and sub-scale scores at $\mathrm{t} 0$ and $\mathrm{t} 1$ ). The intra-subjects WOMAC score changes between $\mathrm{t} 0$ and $\mathrm{t} 1$ were evaluated by the Wilcoxon signed-rank test. A $p$ value $<0.05$ was considered statistically significant.

\section{RESULTS}

Patient characteristics and WOMAC overall and sub-scale scores at t0 and t 1 were listed in Table 1. There were no statistically significant differences in terms of sex prevalence and WOMAC scores (both in $\mathrm{t} 0$ and $\mathrm{t} 1$ ) between $\mathrm{K}-\mathrm{L}$ scale grade 2 and 3 groups. The discrepancy between the median ages of the above mentioned groups of 2.5 years was slightly higher than the $p$ value cut-off considered statistically significant $(p=0.0502)$.

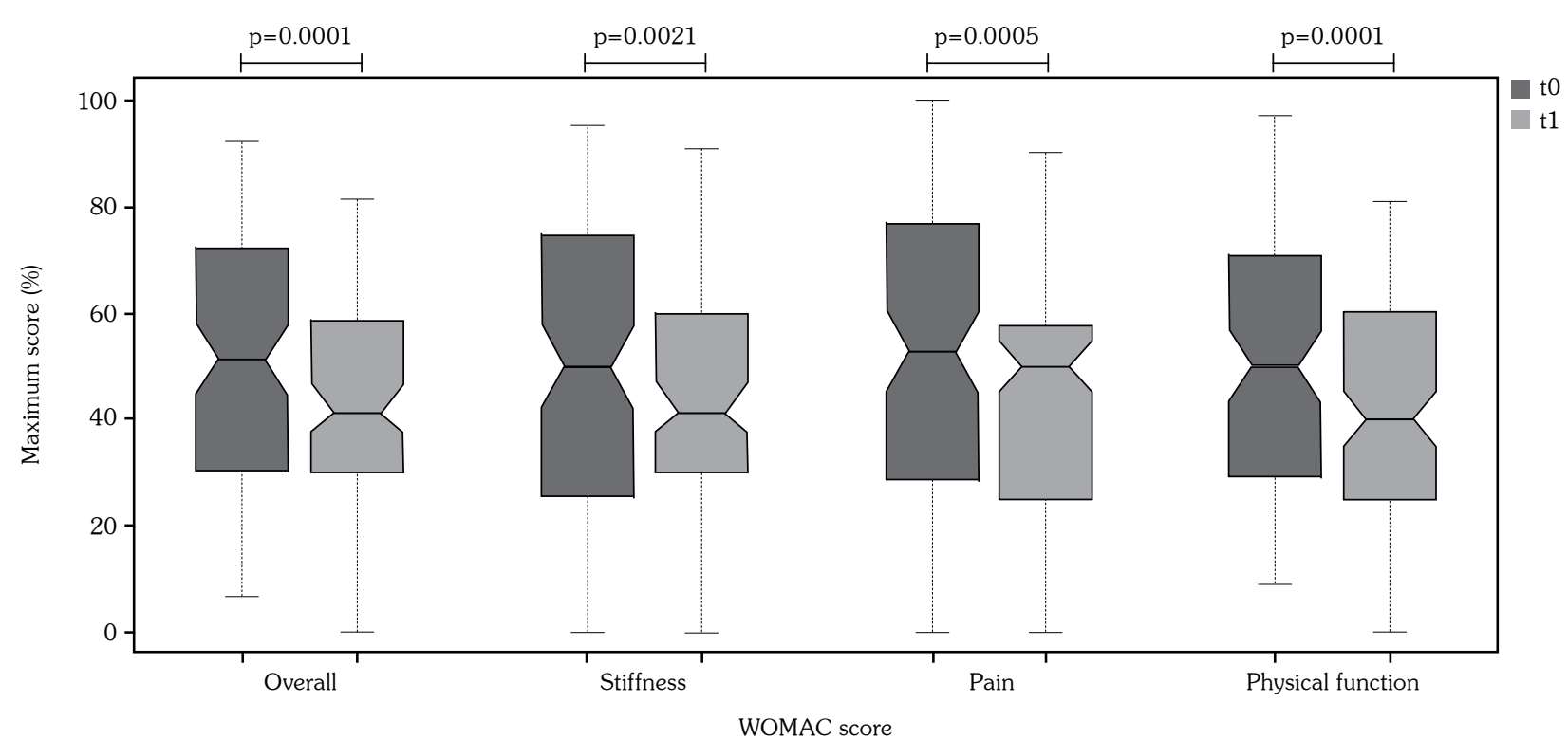

Figure 1. Comparison between WOMAC scores and sub-scale scores before and after treatment with cetylated fatty acid. WOMAC: Western Ontario and McMaster Universities Osteoarthritis Index. 
After one week of CFA application, the whole cohort showed decreased WOMAC overall scores and sub-scale scores (Figure 1) $(p<0.005)$. The score changes in patients' subgroups (K-L grade 2 vs. K-L grade 3 groups) had a similar tendency $(p<0.05)$ : only the stiffness score modification in $\mathrm{K}-\mathrm{L}$ grade 2 group did not reach statistical significance $(p=0.0521)$.

\section{DISCUSSION}

As far as we know, this is the largest cohort of knee OA patients where the effect of CFA topical treatment was investigated. Previously, oral CFA treatment achieved positive outcomes (e.g. knee range of movement, stair climbing ability, static postural stability, and up and go test) in knee OA subjects. ${ }^{8,11,12} \mathrm{Up}$ to date, no standardized protocol for topical CFA cream administration has been designed; however, in general, it appears that tangible advantages are evident after one or two months of continuous application. ${ }^{8,11,12}$ Nevertheless, Kraemer et al. ${ }^{9}$ described WOMAC score improvement with one-week lasting treatment. In accordance with this study, our results showed that one-week CFA cream application (twice per day) appears to be sufficient to verify its effectiveness in early or advanced knee OA.

In this study, we observed an average improvement of 20\% in baseline WOMAC (both overall and sub-scale scores) in patients with early (K-L grade 2) and advanced (K-L grade 3) knee OA. Furthermore, there was a relatively low WOMAC pain score decrease (less than 10\%) in both groups. This outcome may be likely due to the oral analgesic treatment that patients were allowed to continue, without dose increase, during the study. The improvement in stiffness score in K-L grade 2 group was not statically significant even if there was a distinct trend. On the other hand, a remarkable change was shown in the WOMAC functional ability score. Thus, these observations strengthen the hypothesis that disability is more influenced by pain than by knee OA severity. ${ }^{16}$

Topical treatment of CFA was effective in subjects with advanced knee OA since they experienced a statistically significant improvement in all WOMAC sub-scale scores. The only baseline difference between the two K-L grade subgroups was the median age. However, from a statistical and biological point of view, this dissimilarity is not enough to account for these results. In general, CFA cream application was more helpful in the advanced knee OA group.

We are aware that this study has some limitations. First is the lack of a control group with subjects receiving a topical placebo. A comparison between CFA and placebo was not within the aim of this study. Although this issue was already extensively assessed in previous investigations, ${ }^{8,9,12}$ a placebo arm would have allowed to obtain clearer results. The lack of evaluation of CFA positive long-lasting effect may be another limitation. However, from a clinical point of view, despite the general interest of this topic, this limitation may be negligible. CFA cream application is not associated with side effects, ${ }^{10}$ thus it can be safely prescribed as self- and on-demand treatment. Finally, the possible reduction in the analgesics' dose was not assessed. We are confident that this is a minor lack as it is usually taken into account as surrogate or secondary endpoint in randomized control trials.

In conclusion, administration of topical CFA may mitigate most common symptoms in knee OA. Our findings suggest that topical CFA is effective in all knee OA patients with slightly higher evidence for those with advanced disease.

\section{Acknowledgements}

We want to thank Prof. Bellamy for developing WOMAC questionnaire and giving us the possibility to use it in this study.

\section{Declaration of conflicting interests}

The authors declared no conflicts of interest with respect to the authorship and/or publication of this article.

\section{Funding}

The authors received no financial support for the research and/or authorship of this article.

\section{REFERENCES}

1. Murphy L, Schwartz TA, Helmick CG, Renner JB, Tudor G, Koch G, et al. Lifetime risk of symptomatic knee osteoarthritis. Arthritis Rheum 2008;59:1207-13. 
2. Bijlsma JW, Knahr K. Strategies for the prevention and management of osteoarthritis of the hip and knee. Best Pract Res Clin Rheumatol 2007;21:59-76.

3. Litwic A, Edwards MH, Dennison EM, Cooper C. Epidemiology and burden of osteoarthritis. Br Med Bull 2013;105:185-99.

4. McAlindon TE, Bannuru RR, Sullivan MC, Arden NK, Berenbaum F, Bierma-Zeinstra SM, et al. OARSI guidelines for the non-surgical management of knee osteoarthritis. Osteoarthritis Cartilage 2014;22:363-88.

5. Brown GA. AAOS clinical practice guideline: treatment of osteoarthritis of the knee: evidencebased guideline, 2nd edition. J Am Acad Orthop Surg 2013;2:577-9.

6. Puenpatom RA, Victor TW. Increased prevalence of metabolic syndrome in individuals with osteoarthritis: an analysis of NHANES III data. Postgrad Med 2009;121:9-20.

7. Curtis $\mathrm{CL}$, Hughes $\mathrm{CE}$, Flannery $\mathrm{CR}$, Little $\mathrm{CB}$, Harwood JL, Caterson B. n-3 fatty acids specifically modulate catabolic factors involved in articular cartilage degradation. J Biol Chem 2000;275:721-4.

8. Hesslink $\mathrm{R}$ Jr, Armstrong D, Nagendran MV, Sreevatsan S, Barathur R. Cetylated fatty acids improve knee function in patients with osteoarthritis. J Rheumatol 2002;29:1708-12.

9. Kraemer WJ, Ratamess NA, Maresh CM, Anderson JA, Volek JS, Tiberio DP, et al. A cetylated fatty acid topical cream with menthol reduces pain and improves functional performance in individuals with arthritis. J Strength Cond Res 2005;19:475-80.
10. Sharan D, Jacob BN, Ajeesh PS, Bookout JB, Barathur RR. The effect of cetylated fatty esters and physical therapy on myofascial pain syndrome of the neck. J Bodyw Mov Ther 2011;15:363-74.

11. Kraemer WJ, Ratamess NA, Anderson JM, Maresh $\mathrm{CM}$, Tiberio DP, Joyce ME, et al. Effect of a cetylated fatty acid topical cream on functional mobility and quality of life of patients with osteoarthritis. $\mathrm{J}$ Rheumatol 2004;31:767-74.

12. Kraemer WJ, Ratamess NA, Maresh CM, Anderson JA, Tiberio DP, Joyce ME, et al. Effects of treatment with a cetylated fatty acid topical cream on static postural stability and plantar pressure distribution in patients with knee osteoarthritis. J Strength Cond Res 2005;19:115-21.

13. Kellgren JH, Lawrence JS. Radiological assessment of osteo-arthrosis. Ann Rheum Dis 1957;16:494-502.

14. Hochberg MC, Altman RD, Brandt KD, Clark BM, Dieppe PA, Griffin MR, et al. Guidelines for the medical management of osteoarthritis. Part II. Osteoarthritis of the knee. American College of Rheumatology. Arthritis Rheum 1995;38:1541-6.

15. Salaffi F, Leardini G, Canesi B, Mannoni A, Fioravanti A, Caporali R, et al. Reliability and validity of the Western Ontario and McMaster Universities (WOMAC) Osteoarthritis Index in Italian patients with osteoarthritis of the knee. Osteoarthritis Cartilage 2003;11:551-60.

16. Cubukcu D, Sarsan A, Alkan H. Relationships between Pain, Function and Radiographic Findings in Osteoarthritis of the Knee: A Cross-Sectional Study. Arthritis 2012;2012:984060. 